\title{
A Single-center, Single Blinded, Randomized Controlled Trial Protocol of Therapeutic Listening Programme Versus Traditional Music Therapy on Depression and Quality of Life in Institutionalized Adolescents
}

\author{
Gandhi Karunanithi Balaji ${ }^{1}$, Preethi John², Gokulakannan Kandasamy ${ }^{3}$ \\ ${ }^{1,2}$ Chitkara School of health sciences, Chitkara University, Punjab, India, \\ ${ }^{3}$ School of Health and Life Sciences, Teesside University, United Kingdom
}

\begin{abstract}
Introduction: Depression is a typical, constant, and debilitating issue occurring during adolescence and influencing near a fourth of all grown-ups. In adolescents, depression related with impaired social connections, lower educational accomplishment, poor scholarly execution, family and social brokenness, physical sick wellbeing and expanded danger of self-destruction. Music therapy intervention could essentially diminished symptoms of depression, increased cognitive functioning and improved quality of life
\end{abstract}

Objective: To determine the effects of therapeutic listening programme versus traditional music therapy on Depression and Quality of Life in Institutionalized Adolescents.

Method: A single-center, single blinded, two group pretest-posttest randomized controlled trial of 36 institutionalized adolescents with depression will be recruited through the simple random sampling. Recruited institutionalized adolescents will be randomized into one of the two groups namely, therapeutic listening programme group and traditional music therapy group through block randomization. Both the groups will receive their respective interventions for 30 minutes a day, 2 days a week for 8 weeks. Participants in dance group performed, 30 minutes of dance for 3 days/week for 6 weeks. The primary outcome measures will be Children's Depression Inventory (CDI) and Youth Quality of Life Instrument - Short Form (YQOL-SF). Urine test and electroencephalogram (EEG) will be recorded in addition to CDI and YQOL-SF. All the outcome measures will be measured at baseline and 8 weeks post intervention.

Results: Normality of the collected data will be analyzed using Shapiro-Wilk test of normality. Descriptive statistics will be expressed in mean \pm Standard deviation/mean ( $95 \%$ confidence interval) with range and parametric tests/non-parametric tests, paired t-test/Wilcoxon signed rank test and independent t-test/MannWhitney U-test will be used for within and between group comparisons based on normality.

Conclusion: The study outlines the importance of therapeutic listening programme on institutionalized adolescents with depression. Successful completion of this RCT will create evidence on the best treatment options for institutionalized adolescents with depression.

Keywords: Adolescents; depression; music therapy; quality of life; therapeutic listening programme.

\section{Corresponding Author:}

Dr. Balaji Gandhi K.

MPT (Neuro), (PhD), Chitkara School of health sciences, Chitkara university, Punjab, India -140401

e-mail: balaji.gandhi@chitkara.edu.in

\section{Introduction}

More than 264 million of world population are suffering from depression. ${ }^{1}$ Injuries, and Risk Factors Study 2017 (GBD 2017 The worst consequences of depression is suicide which ranks second in leading cause of death among the age groups between 15 and 29 
year. ${ }^{2}$ Depression is a typical, constant, and debilitating issue occurring during adolescence and influencing near a fourth of all grown-ups. Around $21-28 \%$ of youth encountering major depression by the age of 19 years. ${ }^{3}$ World Health Organization evaluates that depression is the third leading cause for worldwide illness, anticipated to be the second cause by 2020 , and will be the first leading causes by $2030 .{ }^{4}$ Depression is related with numerous physical, passionate, mental, and social changes in youngsters. They experience intense emotions and may go through many stressful events in this period. These rapid changes in all aspects of their life affect their mental health and increase the risk of depression.

In adolescents, depression related with impaired social connections, lower educational accomplishment, poor scholarly execution, family and social brokenness, physical sick wellbeing and expanded danger of selfdestruction. ${ }^{5}$ Depression is the significant reasons for morbidity among youths, frequently taking a constant, repetitive, and episodic course. ${ }^{4}$ Major Depression Episode are weigh or appetite changes, sleep difficulties (sleep deprivation or hypersomnia), exhaustion or loss of vitality, psychomotor agitation or impediment, reduced capacity to concentrate, sentiments of uselessness or guilt, and suicidal thoughts. Cognitive dysfunction, age, psychosis, joblessness, self-destruction thoughts are related with severity of depression. ${ }^{6}$ named main criteria. Although the secondary symptoms can be divided into somatic and non-somatic clusters, the DSM-5 identify depression in all or none fashion. In contrast, depression severity is a continuous variable. Therefore, it is commonly assessed with scales such as the Hamilton Depression Rating Scale (HAMD

Bill Ahessy surveyed if investment in music therapy can decrease depressive symptoms and improve quality of life and cognitive functioning in older population. In the music therapy group, depressive symptoms were decreased by $54 \%, 57 \%$ improvement in Quality of life score and there was a critical increment in cognitive functioning. The investigation inferred that music therapy intervention essentially diminished symptoms of depression, increased cognitive functioning and improved quality of life. ${ }^{7}$

One such strategy, Therapeutic Listening ${ }^{\circledR}$ utilizes electronically modified music using highquality headphones. Auditory input adds to arousal, self-guideline, and emotions. ${ }^{8,9}$ Therapeutic Listening created by Sheila Frick in 1990s for people with sensory processing difficulties. ${ }^{10}$ According to advanced brain technologies (ABT) therapeutic listening program (TLP) uses psycho-acoustically modified classical music listened through a set of sennhieser headphones focusing on certain recurrence extends that guarantee to affect functional capabilities. ${ }^{11}$ relating to 28 individual school-based programs, were identified through the Cochrane Library, PsycInfo and PubMed databases. A large proportion of the programs identified were based on cognitive behavioural therapy (CBT It was initially recommended that auditory intervention training (AIT) was gainful for youngsters with listening tuning and attentional troubles.

Therapeutic listening activates the vestibularcochlear framework by means of the vestibular nuclei, and helps to strengthen muscles of middle ear and improving the capacity to tolerate sound. Modulated music exercises and retrains the tensor tympani and stapedius muscles to permit them to contract. The adjusted music is prepared utilizing low pass- high pass channel. In high pass channel, sounds over $1,000 \mathrm{~Hz}$ pass continuous while sounds underneath $1,000 \mathrm{~Hz}$ are quieted. Music triggers centers in the brainstem that are involved in basic sensorimotor modulation and integration where the sound is modulated and just the low and high sounds are sent up to the cortex. AIT animates reticular activating system, role in neuroplasticity, gets information from vestibular system and release neurotransmitters which aid in arousal, learning and emotion. ${ }^{10,12}$

Therapeutic listening programme (TLP) expands on altered music in recurrence zones. These frequencies zones affects the functional capabilities. Zone one lower-recurrence sounds $(0-750 \mathrm{~Hz})$ help with sensory integration which incorporates rhythm, coordination, balance, muscle tone, laterality and right/left discrimination, sense of direction and body awareness. Zone two spotlights on midrange recurrence sounds $(750-4000 \mathrm{~Hz})$ and connected to language and speech development. Abilities in this zone incorporate language, speech, focus, memory, attention and vocal control. Zone three connect to high-recurrence sounds (4000 $\mathrm{Hz}$ or more) intended to improve abilities, for example ideas, energy, spirituality, intuition and creativity., ${ }^{92-15}$

There is a dearth of evidence supporting the use of TLP in people having depression. Literature on therapeutic listening had been limited to children with sensory processing disorders. ${ }^{10,12,16,17}$ implementing. 
The Listening Program(R Till date only conservative and traditional interventions along with music therapy are used to treat depression in adolescent. Hence, we aimed to explore the effect of therapeutic listening on severity of depression and quality of life in institutionalized adolescent. The objectives of our proposed study are to explore the effects of therapeutic listening over traditional music on severity of Depression and quality of life in institutionalized adolescents.

\section{Materials and Method}

Ethical Statement: The study protocol for the Single blinded Randomized Controlled Trial was approved by the institutional ethics committee and the study will be performed according to the principles laid by, declaration of Helsinki (Revised 2013), Council for International Organizations of Medical Sciences (CIOMS) guidelines, International ethical guidelines for health-related research involving humans (2016) and National guidelines for biomedical and health research involving human participants by Indian council of Medical Research (ICMR), 2017. The ethical guidelines that followed the national ethical guidelines for biomedical and health research involving human participants by Indian council of Medical Research (ICMR), 2017. Consent for participation in the study will be obtained from the participants and their parents/ guardian prior to the start of the study.

Recruitment: Qualified therapist will screen the institutionalized individuals and recruit the study participants based on the inclusion and exclusion criteria displayed below as the selection criteria.

\section{Selection Criteria:}

\section{Inclusion Criteria:}

- Institutionalized Adolescent boys and Girls (age 1218 years)

- Participants Assessed by Children depression Inventory (having average/lower, high average and elevated)

\section{Exclusion Criteria:}

- Presence of any other psychiatric disorder (Schizophrenia, Bipolar Disorder, Anxiety Disorder etc).

- Patients with Hearing impairment

- Patients with untreated active ear infection
After screening, the participants will be recruited thirty six samples by the simple random sampling method from NGO (Gur aasra, Mohali \& Prabh Aasra, Kurali). After obtaining the consent, their demographics will be recorded.

Randomization: Total recruited sample size 36 will be randomly allocated by Block Randomization method by SNOSE (Sequentially numbered opaque sealed envelope) method by Principle investigator into one of the two groups, therapeutic listening programme group (TLPG) and traditional music therapy group (TMTG). The blocks will be in even numbers and the matrix will be $4 \times 9$ (36). There will be total 4 blocks and 9 rows. Then subjects will be allocated in the blocks by using random allocation sequence. Randomization will be done by $4 \mathrm{X}$ 9 matrix design. There will be random blocks of 4 , with each block randomizing 4 participants into TLPG and TMTG each with two participants. The participants will be allotted to group based on the generated randomized sequence. Once the block will be allotted next row block sequence will be opened for the recruitment. Thus equal number of participants will be assigned to both the group overtime. The outcome assessor will be blinded and hence, the randomized controlled trial will be single blinded.

Study Intervention: Intervention will be administered by an experienced physiotherapist who is certified and trained in therapeutic listening programme. Participants in the experimental group will receive therapeutic listening therapy for the duration of $30 \mathrm{~min}$ per session twice a day for 5 days a week for 8 weeks. Detailed week wise therapeutic listening protocol is listed below in Table 1. The sound to be used will be of rhythm and Rhyme, Peach Jamz, Nature Winds, Mozart for modulation and Strawberry Jamz, Vivaldi for modulation. Traditional music therapy group will receive traditional music therapy intervention using earphones with duration of 2 sessions of 30 minutes each per day, 5 days a week for 8 weeks. Intervention will be delivered in a quiet room using Headphones - High-quality Sennheiser headphones which has high resistanceimpedance with a minimum of $150 \mathrm{ohms}$ and 23,000 Hz frequency sensitivity. Therapist will be maintaining the treatment record of each participants. Post intervention evaluation of the outcomes will be measured by using Children's Depression Inventory (CDI), Youth Quality of Life Instrument - Short Form (YQOL-SF), Urine test and EEG. If the participants develops unexpected seizures or syncope during the ongoing therapeutic listening, the study will be discontinued. 
Table 1: Week wise proposed intervention for the therapeutic listening program

\begin{tabular}{|c|c|c|c|c|c|}
\hline S.No. & Total Duration & Mode & Weeks & Album Title & Dose \\
\hline \multirow{4}{*}{1.} & \multirow{4}{*}{4 Weeks } & \multirow{4}{*}{ Engagement } & Week 1 & Rhythm \& Rhyme & \multirow{4}{*}{$\begin{array}{l}30 \text { min per session twice a } \\
\text { day for } 5 \text { days a week }\end{array}$} \\
\hline & & & Week 2 & Peach Jamz & \\
\hline & & & Week 3 & Nature winds & \\
\hline & & & Week 4 & Mozart for modulation & \\
\hline \multirow{2}{*}{2.} & \multirow{2}{*}{2 Weeks } & \multirow{2}{*}{ Interaction } & Week 5 & Strawberry Jamz & \multirow{2}{*}{$\begin{array}{l}30 \text { min per session twice a } \\
\text { day for } 5 \text { days a week }\end{array}$} \\
\hline & & & Week 6 & Vivaldi for Modulation & \\
\hline 3. & 2 Weeks & Discrimination & Week 7 \& Week 8 & $\begin{array}{l}\text { More Mozart for } \\
\text { modulation }\end{array}$ & $\begin{array}{l}30 \text { min per session twice a } \\
\text { day for } 5 \text { days a week }\end{array}$ \\
\hline
\end{tabular}

\section{Outcome Measures:}

Children's Depression Inventory (CDI): The Children's Depression Inventory (CDI) was developed by Kovacs and Beck in 1977. ${ }^{18}$ From Beck's Depression Inventory as a downward revision, CDI was developed originally. This is the commonly used depression measures to assess the severity of depression among the children between 7 and 17 years. CDI has 27 items and takes approximately 10 to 15 minutes to complete the test. CDI has acceptable reliability and validity with other psychometric properties. ${ }^{19,20}$

Youth Quality of Life Instrument-Short Form (YQOL-SF): Youth Quality of Life Instrument - Short Form (YQOL-SF) derived from 41-item instrument through Rasch methodology, Youth Quality of life developed to measure the quality of life among youth aged 12 to 18 years. ${ }^{21,22}$ YQOL-SF has 16 items and four domains to measure, self, social relationships, environment, and general quality of life. Psychometric properties of YQOL-SF is acceptable to measure quality of life among the youth with and without chronic disease and disabilities. $^{23,24}$

Other Outcome Measures: Urine test will be performed to check the levels of Serotonin, cortisol, testosterone, estrogen and electroencephalogram (EEG) to measure the electrical activity of brain to music.

All the outcome measures will be measured at baseline and 8 weeks post intervention.

\section{Results and Discussion}

Normality of the collected data will be analyzed using Shapiro-Wilk test as the sample size is less than 50 . If the data follows normal distribution, then descriptive statistics will be expressed in mean \pm Standard deviation and parametric tests, paired t-test and independent $t$-test will be used for within and between group comparisons. On contrary, if the data does not follow normal distribution, then descriptive statistics will be expressed in mean (95\% confidence interval) with range and nonparametric tests, Wilcoxon signed rank test and MannWhitney U-test will be used for within and between group comparisons. For all the statistical analysis, $\mathrm{p}<0.05$ will be set as significant.

This will be the first RCT to be conducted to gain deeper understanding on the effect of therapeutic listening technique on depression and outlines the importance of therapeutic listening programme on institutionalized adolescents with depression. Successful completion of this RCT will create high level of evidence on the best treatment options for institutionalized adolescents with depression.

\section{Conclusion}

The feasibility of implementing therapeutic listening programme will be tested by the end of this trial and therapeutic listening programme could be an adjunct tool along with exercises to decreases the depressive symptoms in adolescent.

Author(s) Contribution: GKB participated in the conception, design, search, and statistical analysis plan of the research data, writing of the scientific article and forwarding of the scientific article. Preethi John and Gokulakannan Kandasamy participated in the conception, design, and plan for statistical analysis of research data and critical review, and forwarding of the scientific article. 
Conflict of Interest: None of the authors have competing interest declared

Funding: No funding

\section{References}

1. James SL, Abate D, Abate KH, Abay SM, Abbafati $\mathrm{C}$, Abbasi N, et al. Global, regional, and national incidence, prevalence, and years lived with disability for 354 diseases and injuries for 195 countries and territories, 1990-2017: a systematic analysis for the Global Burden of Disease Study 2017. Lancet [Internet]. 2018 Nov 10 [cited 2020 Oct 2];392(10159):1789-858. Available from: https://linkinghub.elsevier.com/retrieve/pii/ S0140673618322797

2. Depression [Internet]. [cited 2020 Oct 2]. Available from: https://www.who.int/news-room/fact-sheets/ detail/depression

3. Mullen S. Major depressive disorder in children and adolescents. Ment Heal Clin. 2018 Nov;8(6):27583.

4. Garber J, Clarke GN, Weersing VR, Beardslee WR, Brent DA, Gladstone TRG, et al. Prevention of depression in at-risk adolescents: a randomized controlled trial. JAMA. 2009 Jun;301(21):221524.

5. Thapar A, Collishaw S, Pine DS, Thapar AK. Depression in adolescence. Lancet (London, England). 2012 Mar;379(9820):1056-67.

6. Tolentino JC, Schmidt SL. DSM-5 Criteria and Depression Severity: Implications for Clinical Practice. Front psychiatry. 2018;9:450.

7. Ahessy B. The use of a music therapy choir to reduce depression and improve quality of life in older adults - A randomized control trial. Music Med. 2016;8(1):17-28.

8. Therapeutic Listening Evidence Brief [Internet]. [cited 2020 Oct 2]. Available from: www.vitallinks. net

9. Preto S, Malloy A, Luong B. Efficacy of Therapeutic Listening ${ }^{\circledR}$ Quickshifts in Children with Efficacy of Therapeutic Listening ${ }^{\circledR}$ Quickshifts in Children with Sensory Processing Difficulties [Internet]. 2017 [cited 2020 Oct 2]. Available from: https:// doi.org/10.33015/dominican.edu/2017.OT.01

10. Gee BM, Thompson K, St John H. Efficacy of a sound-based intervention with a child with an autism spectrum disorder and auditory sensory overresponsivity. Occup Ther Int. 2014 Mar;21(1):1220.

11. Calear AL, Christensen H. Systematic review of school-based prevention and early intervention programs for depression. J Adolesc. 2010 Jun;33(3):429-38.

12. Villasenor RF, Smith SL, Jewell VD. A systematic review of sound-based intervention programs to improve participation in education for children with sensory processing and integration challenges. J Occup Ther Sch Early Interv [Internet]. 2018;11(2):172-91. Available from: https://doi.or $\mathrm{g} / 10.1080 / 19411243.2018 .1432444$

13. Abbott R. A study on the effects of therapeutic listening on a study on the effects of therapeutic listening on the social and functional behavior of preschool the social and functional behavior of preschool children with autism spectrum disorder children with autism spectrum disorder [Internet]. [cited 2020 May 26]. Available from: https:// scholarscompass.vcu.edu/etd/2620

14. Tashjian H, Hair D, Taasan P. Measuring The Outcomes of Therapeutic Listening in Children with Learning and Developmental Disabilities [Internet]. Graduate Master's Theses, Capstones, and Culminating Projects. [San Rafael, California]: Dominican University of California; 2018 [cited 2020 May 26]. Available from: https://scholar. dominican.edu/masters-theses/292

15. Wink S, McKeown L, Casey J. Parents' perspectives of using a therapeutic listening program with their children with sensory processing difficulties: A qualitative study. J Occup Ther Sch Early Interv [Internet]. 2017;10(2):147-70. Available from: https://doi.org/10.1080/19411243.2017.1304839

16. Randell E, McNamara R, Delport S, Busse M, Hastings RP, Gillespie D, et al. Sensory integration therapy versus usual care for sensory processing difficulties in autism spectrum disorder in children: study protocol for a pragmatic randomised controlled trial. Trials. 2019 Feb;20(1):113.

17. Zimmer M, Desch L. Sensory integration therapies for children with developmental and behavioral disorders. Pediatrics. 2012 Jun;129(6):1186-9.

18. Kovacs M. The Children's Depression, Inventory (CDI). Psychopharmacol Bull. 1985;21(4):995-8.

19. Hodges KK, Siegel LJ, Mullins L, Griffin N. Factor 
analysis of the Children's Depression Inventory. Psychol Rep. 1983 Dec;53(3 Pt 1):759-63.

20. Saylor CF, Finch AJJ, Spirito A, Bennett B. The children's depression inventory: a systematic evaluation of psychometric properties. J Consult Clin Psychol. 1984 Dec;52(6):955-67.

21. Edwards TC, Huebner CE, Connell FA, Patrick DL. Adolescent quality of life, part I: conceptual and measurement model. J Adolesc. 2002 Jun;25(3):275-86.

22. Patrick DL, Edwards TC, Topolski TD. Adolescent quality of life, part II: initial validation of a new instrument. J Adolesc. 2002 Jun;25(3):287-300.
23. Michalos AC, editor. YQOL-SF. In: Encyclopedia of Quality of Life and Well-Being Research [Internet]. Dordrecht: Springer Netherlands; 2014. p. 7309. Available from: https://doi. org/10.1007/978-94-007-0753-5_104622

24. Patrick DL, Edwards TC, Skalicky AM, Schick B, Topolski TD, Kushalnagar P, et al. Validation of a quality-of-life measure for deaf or hard of hearing youth. Otolaryngol neck Surg Off J Am Acad Otolaryngol Neck Surg. 2011 Jul;145(1):137-45. 